\title{
HIGHER ORDER FUNCTIONAL BOUNDARY VALUE PROBLEMS: EXISTENCE AND LOCATION RESULTS
}

\author{
JOHN R. GRAEF, LINGJU KONG, AND FELIZ M. MINHÓS ${ }^{1}$
}

Abstract. This paper considers the $n^{\text {th }}$-order boundary value problem consisting of the equation

$$
-\left(\phi\left(u^{(n-1)}(x)\right)\right)^{\prime}=f\left(x, u(x), \ldots, u^{(n-1)}(x)\right), \quad x \in(0,1),
$$

together with the boundary conditions

$$
\begin{aligned}
& g_{i}\left(u, u^{\prime}, \ldots, u^{(n-2)}, u^{(i)}(0)\right)=0, \quad i=0, \ldots, n-3, \\
& g_{n-2}\left(u, u^{\prime}, \ldots, u^{(n-2)}, u^{(n-2)}(0), u^{(n-1)}(0)\right)=0, \\
& g_{n-1}\left(u, u^{\prime}, \ldots, u^{(n-2)}, u^{(n-2)}(1), u^{(n-1)}(1)\right)=0,
\end{aligned}
$$

where $\phi$ is an increasing homeomorphism such that $\phi(0)=0, n \geq 2$ is an integer, $I:=[0,1]$, and $f: I \times \mathbb{R}^{n} \rightarrow \mathbb{R}$ is an $L^{1}$-Carathéodory function. Here, $g_{i}:(C(I))^{n-1} \times \mathbb{R} \rightarrow \mathbb{R}$, $i=0, \ldots, n-3$, and $g_{n-2}, g_{n-1}:(C(I))^{n-1} \times \mathbb{R}^{2} \rightarrow \mathbb{R}$ are continuous functions satisfying certain monotonicity assumptions. We present sufficient conditions on the nonlinearity and the boundary conditions to ensure the existence of solutions. Moreover, from the lower and upper solutions method, some information is given about the location of the solution and its qualitative properties. Due to the functional dependence in the boundary conditions, this work generalizes several results for higher order problems with many types of boundary conditions. The main results are illustrated with examples.

\section{INTRODUCTION}

Consider the $n^{\text {th }}$-order differential equation

$$
-\left(\phi\left(u^{(n-1)}(x)\right)\right)^{\prime}=f\left(x, u(x), \ldots, u^{(n-1)}(x)\right), \quad x \in(0,1)
$$

with the boundary conditions

$$
\begin{aligned}
& g_{i}\left(u, u^{\prime}, \ldots, u^{(n-2)}, u^{(i)}(0)\right)=0, \quad i=0, \ldots, n-3, \\
& g_{n-2}\left(u, u^{\prime}, \ldots, u^{(n-2)}, u^{(n-2)}(0), u^{(n-1)}(0)\right)=0, \\
& g_{n-1}\left(u, u^{\prime}, \ldots, u^{(n-2)}, u^{(n-2)}(1), u^{(n-1)}(1)\right)=0,
\end{aligned}
$$

where $\phi$ is an increasing homeomorphism such that $\phi(0)=0, n \geq 2$ is an integer, $I:=[0,1]$, $f: I \times \mathbb{R}^{n} \rightarrow \mathbb{R}$ is a $L^{1}$-Carathéodory function, and $g_{i}:(C(I))^{n-1} \times \mathbb{R} \rightarrow \mathbb{R}, i=0, \ldots, n-3$,

\footnotetext{
${ }^{1}$ Partially supported by Fundação para a Ciência e Tecnologia (FCT), Project SFRH/BSAB/849/2008.
} 
and $g_{n-2}, g_{n-1}:(C(I))^{n-1} \times \mathbb{R}^{2} \rightarrow \mathbb{R}$ are continuous functions satisfying certain monotonicity assumptions that will be described below.

Not only do these types of fully higher order equations include a large range of differential equations, but the functional dependence on the derivatives up to order $n-2$ in the boundary conditions allows the application of the problem (1), (2) to many boundary value problems (BVPs) involving real world phenomena. As examples of recent results on BVPs, we refer the reader to $[6,8,11]$ for two-point problems, $[4,5,7,9,10,14]$ for multipoint cases, and to $[1,2,3,12]$ for functional problems.

The arguments used in this work are motivated by those in [5] and are based on a priori estimates of the possible solutions $u$. A Nagumo-type condition plays an important role in bounding $u^{(n-1)}$. A fixed-point result and the lower and upper solution method are used to obtain the existence and location of the solution and its derivatives up to order $n-2$. The difficulties raised by the functional part of the boundary conditions are overcome with a truncation technique. We are also able to avoid the usual assumption on $\phi$ found in the literature, namely, $\phi(\mathbb{R})=\mathbb{R}$.

We point out that in our main result (Theorem 6 below), the assumption on the nonlinearity $f$ is weaker than the monotone conditions generally assumed in the literature. In fact, we only assume (see (14) below) that some growth condition is satisfied in a set defined by some appropriate lower and upper solutions.

\section{Definitions And Auxiliary Results}

For the reader's convenience, this section summarizes some definitions and results to be used in the remainder of the paper. Let $L^{p}, 1 \leq p \leq \infty$, be the usual spaces with the 
usual norms

$$
\|u\|_{p}= \begin{cases}\left(\int_{0}^{1}|u(t)|^{p} d t\right)^{1 / p}, & 1 \leq p<\infty, \\ \sup \{|u(t)|: t \in I\}, & p=\infty\end{cases}
$$

The function $f: I \times \mathbb{R}^{n} \rightarrow \mathbb{R}$ is said to be a Carathéodory function if it satisfies the following conditions:

(i) For each $y \in \mathbb{R}^{n}$, the function $f(\cdot, y)$ is measurable on $I$;

(ii) For a. e. $x \in I$, the function $f(x, \cdot)$ is continuous on $\mathbb{R}^{n}$;

(iii) For each compact set $K \subset \mathbb{R}^{n}$ there is a function $m_{K} \in L^{1}(I)$ such that

$$
|f(x, y)| \leq m_{K}(x) \quad \text { for a.e. } \quad x \in I \quad \text { and all } \quad y \in K \text {. }
$$

The Nagumo-type growth condition defined next will allow us to find an a priori estimate on the derivative $u^{(n-1)}$ of a solution $u$.

Definition 1. Given a subset $E \subset I \times \mathbb{R}^{n}$, a function $f: I \times \mathbb{R}^{n} \rightarrow \mathbb{R}$ satisfies a Nagumo-type condition in the set

$$
E:=\left\{\left(x, y_{0}, \ldots, y_{n-1}\right) \in I \times \mathbb{R}^{n}: \gamma_{j}(x) \leq y_{j} \leq \Gamma_{j}(x), j=0, \ldots, n-2\right\}
$$

with $\gamma_{j}, \Gamma_{j} \in C(I, \mathbb{R})$ and

$$
\gamma_{j}(x) \leq \Gamma_{j}(x) \text { for all } x \in I, j=0, \ldots, n-2 \text {, }
$$

if there exists $h_{E} \in L^{1}(0,1)$ and $R>r$, where

$$
r:=\max \left\{\Gamma_{n-2}(1)-\gamma_{n-2}(0), \Gamma_{n-2}(0)-\gamma_{n-2}(1)\right\}
$$

such that

$$
\left|f\left(x, y_{0}, \ldots, y_{n-1}\right)\right| \leq h_{E}\left(\left|y_{n-1}\right|\right) \text { for all }\left(x, y_{0}, \ldots, y_{n-1}\right) \in E
$$

and

$$
\int_{\phi(r)}^{\phi(R)} \frac{\left|\phi^{-1}(s)\right|}{h_{E}\left(\left|\phi^{-1}(s)\right|\right)} d s>\max _{x \in I} \Gamma_{n-2}(x)-\min _{x \in I} \gamma_{n-2}(x) .
$$

The a priori bound mentioned above is given by next result. 
Lemma 2. ([6, Lemma 2]) Suppose $\gamma_{j}, \Gamma_{j} \in C(I, \mathbb{R})$ are such that

$$
\gamma_{j}(x) \leq \Gamma_{j}(x) \text { for all } x \in I, j=0, \ldots, n-2 \text {, }
$$

and let $f: E \rightarrow \mathbb{R}$ be a Carathéodory function satisfying a Nagumo-type condition in $E$. Then there exists $R>0$ (depending only on $\gamma_{n-2}, \Gamma_{n-2}$, and $h_{E}$ ) such that every solution $u(x)$ of (1) with

$$
\gamma_{j}(x) \leq u^{(j)}(x) \leq \Gamma_{j}(x) \text { for all } x \in I, j=0, \ldots, n-2
$$

satisfies $\left\|u^{(n-1)}\right\|_{\infty}<R$.

The next lemma gives the existence and uniqueness of solutions for a problem related to (1)-(2) above.

Lemma 3. ([6, Lemma 3]) Let $\varphi: \mathbb{R} \rightarrow \mathbb{R}$ be an increasing homeomorphism such that $\varphi(0)=0$ and $\varphi(\mathbb{R})=\mathbb{R}$, let $p: I \rightarrow \mathbb{R}$ satisfy $p \in L^{1}(I)$, and let $A_{i}, i=0, \ldots, n-3, B_{1}$, $C_{1} \in \mathbb{R}$. Then the problem

$$
\left\{\begin{array}{c}
-\left(\varphi\left(u^{(n-1)}(x)\right)\right)^{\prime}=p(x), \quad \text { for a.e. } x \in I, \\
u^{(i)}(0)=A_{i}, \quad i=0, \ldots, n-3, \\
u^{(n-2)}(0)=B_{1}, \\
u^{(n-2)}(1)=C_{1},
\end{array}\right.
$$

has a unique solution given by

$$
u(x)=B_{1}+\int_{0}^{x} \varphi^{-1}\left(\tau_{v}-\int_{0}^{s} p(r) d r\right) d s
$$

if $n=2$, and

$$
u(x)=\sum_{k=0}^{n-3} A_{k} \frac{x^{k}}{k !}+\int_{0}^{x} \frac{(x-r)^{n-3}}{(n-3) !} v(r) d r
$$

if $n \geq 3$, where

$$
v(x):=B_{1}+\int_{0}^{x} \varphi^{-1}\left(\tau_{v}-\int_{0}^{s} p(r) d r\right) d s
$$


and $\tau_{v} \in \mathbb{R}$ is the unique solution of the equation

$$
C_{1}-B_{1}=\int_{0}^{1} \varphi^{-1}\left(\tau_{v}-\int_{0}^{s} p(r) d r\right) d s
$$

Some properties of truncated functions that will be used in our proofs are given in the following result.

Lemma 4. ([13, Lemma 2]) For $z, w \in C(I)$ such that $z(x) \leq w(x)$, for every $x \in I$, define

$$
q(x, u)=\max \{z, \min \{u, w\}\}
$$

Then, for each $u \in C^{1}(I)$, the following two properties hold:

(a) $\frac{d}{d x} q(x, u(x))$ exists for a.e. $x \in I$;

(b) If $u, u_{m} \in C^{1}(I)$ and $u_{m} \rightarrow u$ in $C^{1}(I)$, then

$$
\frac{d}{d x} q\left(x, u_{m}(x)\right) \rightarrow \frac{d}{d x} q(x, u(x)) \text { for a.e. } x \in I .
$$

The functions used as lower and upper solutions are defined as follows. Here, $A C(I)$ denotes the set of absolutely continuous functions on $I$.

Definition 5. Let $n \geq 2$. A function $\alpha \in C^{n-1}(I)$ such that $\phi\left(\alpha^{(n-1)}(x)\right) \in A C(I)$ is a lower solution of the problem (1)-(2) if

$$
-\left(\phi\left(\alpha^{(n-1)}(x)\right)\right)^{\prime} \leq f\left(x, \alpha(x), \alpha^{\prime}(x), \ldots, \alpha^{(n-1)}(x)\right)
$$

for $x \in(0,1)$, and

$$
\begin{aligned}
& g_{i}\left(\alpha, \alpha^{\prime}, \ldots, \alpha^{(n-2)}, \alpha^{(i)}(0)\right) \geq 0, \quad i=0, \ldots, n-3, \\
& g_{n-2}\left(\alpha, \alpha^{\prime}, \ldots, \alpha^{(n-2)}, \alpha^{(n-2)}(0), \alpha^{(n-1)}(0)\right) \geq 0, \\
& g_{n-1}\left(\alpha, \alpha^{\prime}, \ldots, \alpha^{(n-2)}, \alpha^{(n-2)}(1), \alpha^{(n-1)}(1)\right) \geq 0,
\end{aligned}
$$

A function $\beta \in C^{n-1}(I)$ such that $\phi\left(\beta^{(n-1)}(x)\right) \in A C(I)$ is an upper solution of the problem (1)-(2) if the reversed inequalities hold. 


\section{Existence AND LOCATION THEOREM}

Our main result is an existence and location theorem, as is usual when the lower and upper solution technique is used in proofs. In this case, some information on the location of the derivatives up to order $n-2$ are also given.

Theorem 6. Let $f: I \times \mathbb{R}^{n} \rightarrow \mathbb{R}$ be a $L^{1}$-Carathéodory function.

Assume that $\alpha$ and $\beta$ are lower and upper solutions of problem (1)-(2), respectively, such that

$$
\begin{aligned}
\alpha^{(n-2)}(x) & \leq \beta^{(n-2)}(x) \quad \text { for all } x \in I, \\
\alpha^{(i)}(0) & \leq \beta^{(i)}(0), i=0, \ldots, n-3
\end{aligned}
$$

$f$ satisfies a Nagumo-type condition (4) in the set

$$
E_{*}=\left\{\left(x, y_{0}, \ldots, y_{n-1}\right) \in I \times \mathbb{R}^{n}: \alpha^{(i)}(x) \leq y_{i} \leq \beta^{(i)}(x), i=0, \ldots, n-2\right\}
$$

and

$$
\begin{aligned}
f\left(x, \alpha(x), \ldots, \alpha^{(n-3)}(x), y_{n-2}, y_{n-1}\right) & \leq f\left(x, y_{0}, \ldots, y_{n-1}\right) \\
& \leq f\left(x, \beta(x), \ldots, \beta^{(n-3)}(x), y_{n-2}, y_{n-1}\right)
\end{aligned}
$$

for fixed $x, y_{n-2}, y_{n-1}$ and $\alpha^{(i)}(x) \leq y_{i} \leq \beta^{(i)}(x), i=0, \ldots, n-3$, for all $x \in I$. Assume that the functions $g_{j}: \mathbb{R} \rightarrow \mathbb{R}, j=0, \ldots, n-1$, are continuous and satisfy:

$g_{i}\left(y_{0}, \ldots, y_{n-1}\right), i=0, \ldots, n-3$, are nondecreasing in $y_{0}, \ldots, y_{n-2}$;

$g_{n-2}\left(y_{0}, \ldots, y_{n-1}, y_{n}\right)$ is nondecreasing in $y_{0}, \ldots, y_{n-2}$ and $y_{n}$;

$g_{n-1}\left(y_{0}, \ldots, y_{n-1}, y_{n}\right)$ is nondecreasing in $y_{0}, \ldots, y_{n-2}$ and nonincreasing in $y_{n}$.

Then problem (1)-(2) has at least one solution u such that

$$
\alpha^{(i)}(x) \leq u^{(i)}(x) \leq \beta^{(i)}(x)
$$


for every $x \in I$ and $i=0, \ldots, n-2$, and

$$
-R \leq u^{(n-1)}(x) \leq R
$$

for some $R>0$.

Remark 7. The relations $\alpha^{(j)}(x) \leq \beta^{(j)}(x), j=0, \ldots, n-3$, are obtained from (12) by integrating and applying the boundary data (13).

Proof. For $i=0, \ldots, n-2$, define the continuous truncations

$$
\delta_{i}(x, w)=\left\{\begin{array}{cccc}
\beta^{(i)}(x) & \text { if } & w>\beta^{(i)}(x) \\
w & \text { if } & \alpha^{(i)}(x) \leq w \leq \beta^{(i)}(x) \\
\alpha^{(i)}(x) & \text { if } & w<\alpha^{(i)}(x)
\end{array}\right.
$$

For

$$
R>\max \left\{\beta^{(n-2)}(1)-\alpha^{(n-2)}(0), \beta^{(n-2)}(0)-\alpha^{(n-2)}(1),\left\|\alpha^{(n-1)}\right\|_{\infty},\left\|\beta^{(n-1)}\right\|_{\infty}\right\}
$$

(see Definition 1), consider the functions

$$
\xi(y)=\max \{-R, \min \{y, R\}\}
$$

and $\varphi: \mathbb{R} \rightarrow \mathbb{R}$ given by

$$
\varphi(y)=\left\{\begin{array}{ccc}
\phi(y), & \text { if } \quad|y| \leq R, \\
\frac{\phi(R)-\phi(-R)}{2 R} y+\frac{\phi(R)+\phi(-R)}{2}, & \text { if } & |y|>R .
\end{array}\right.
$$

Define the modified problem composed of the differential equation

$$
\begin{aligned}
& -\left(\varphi\left(u^{(n-1)}(x)\right)\right)^{\prime} \\
= & f\left(x, \delta_{0}(x, u), \ldots, \delta_{n-2}\left(x, u^{(n-2)}\right), \xi\left(\frac{d}{d x} \delta_{n-2}\left(x, u^{(n-2)}\right)\right)\right) \equiv F_{u}(x)
\end{aligned}
$$

and the boundary conditions

$$
\begin{aligned}
u^{(i)}(0) & =\delta_{i}\left(0, u^{(i)}(0)+g_{i}\left(u, \ldots, u^{(n-2)}, u^{(i)}(0)\right)\right), i=0, \ldots, n-3, \\
u^{(n-2)}(0) & =\delta_{n-2}\left(0, u^{(n-2)}(0)+g_{n-2}\left(u, \ldots, u^{(n-2)}, u^{(n-2)}(0), u^{(n-1)}(0)\right)\right), \\
u^{(n-2)}(1) & =\delta_{n-2}\left(1, u^{(n-2)}(1)+g_{n-1}\left(u, \ldots, u^{(n-2)}, u^{(n-2)}(1), u^{(n-1)}(1)\right)\right) .
\end{aligned}
$$


A function $u \in C^{n-1}(I)$ such that $\varphi \circ u^{(n-1)} \in A C(I)$ is a solution of problem (18)-(19) if it satisfies the above equalities.

Step 1: Every solution of problem (18)-(19) satisfies

$$
\begin{aligned}
\alpha^{(i)}(x) & \leq u^{(i)}(x) \leq \beta^{(i)}(x) \quad \text { for } \quad i=0, \ldots, n-2 \\
-R & \leq u^{(n-1)}(x) \leq R
\end{aligned}
$$

in $I$.

For a solution $u$ of (18)-(19), assume, for the sake of a contradiction, that the right hand inequality in (20) does not hold for $i=n-2$, and define

$$
\max _{x \in I}(u-\beta)^{(n-2)}(x):=(u-\beta)^{(n-2)}\left(x_{0}\right)>0 .
$$

By $(19), u^{(n-2)}(0) \leq \beta^{(n-2)}(0)$ and $u^{(n-2)}(1) \leq \beta^{(n-2)}(1)$. So, $x_{0} \in(0,1), u^{(n-1)}\left(x_{0}\right)=$ $\beta^{(n-1)}\left(x_{0}\right)$, and there is $\varepsilon>0$ such that

$$
u^{(n-2)}\left(x_{0}+\varepsilon\right)=\beta^{(n-2)}\left(x_{0}+\varepsilon\right)
$$

and $u^{(n-2)}(x)>\beta^{(n-2)}(x)$ on $\left[x_{0}, x_{0}+\varepsilon\right)$.

On $\left(x_{0}, x_{0}+\varepsilon\right)$, by Definition $5,(14),(15),(16)$, and (17), we have

$$
\begin{aligned}
& -\left(\varphi\left(u^{(n-1)}(x)\right)\right)^{\prime} \\
= & f\left(x, \delta_{0}(x, u), \ldots, \delta_{n-2}\left(x, u^{(n-2)}\right), \xi\left(\frac{d}{d x} \delta_{n-2}\left(x, u^{(n-2)}\right)\right)\right) \\
= & f\left(x, \delta_{0}(x, u), \ldots, \delta_{n-3}\left(x, u^{(n-3)}\right), \beta^{(n-2)}(x), \beta^{(n-1)}(x)\right) \\
\leq & f\left(x, \beta(x), \ldots, \beta^{(n-3)}, \beta^{(n-2)}(x), \beta^{(n-1)}(x)\right) \\
\leq & -\left(\phi\left(\beta^{(n-1)}(x)\right)\right)^{\prime}=-\left(\varphi\left(\beta^{(n-1)}(x)\right)\right)^{\prime} .
\end{aligned}
$$

Therefore, $u^{(n-1)}(x) \geq \beta^{(n-1)}(x)$ on $\left(x_{0}, x_{0}+\varepsilon\right)$, which is a contradiction to the definition of $\left[x_{0}, x_{0}+\varepsilon\right)$. So $u^{(n-2)}(x) \leq \beta^{(n-2)}(x)$ for every $x \in I$. By analogous arguments, it can be shown that $\alpha^{(n-2)}(x) \leq u^{(n-2)}(x)$ in $I$. 
Integrating the inequalities

$$
\alpha^{(n-2)}(x) \leq u^{(n-2)}(x) \leq \beta^{(n-2)}(x)
$$

in $[0, x]$, and using (13) and (19), we see that (20) holds for $i=0, \ldots, n-2$.

From Lemma 4 and the definition of $\xi$, the right hand side of equation (18) is a $L^{1}$ function. Therefore, Lemma 2 can be applied, with $\gamma_{j}(x)=\alpha^{(j)}(x)$ and $\Gamma_{j}(x)=\beta^{(j)}(x)$, for $j=0, \ldots, n-2$, that is, $(21)$ holds.

Step 2: Problem (18)-(19) has a solution $u_{1}(x)$.

Let $u \in C^{n-1}(I)$ be fixed. If $n \geq 3$, by Lemma 3, solutions of (18)-(19) are the fixed points of the operator

$$
\begin{aligned}
\mathcal{T} u(x)= & \sum_{k=0}^{n-3} \delta_{k}\left(0, u^{(i)}(0)+g_{k}\left(u, u^{\prime}, \ldots, u^{(n-2)}, u^{(i)}(0)\right)\right) \frac{x^{k}}{k !} \\
& +\int_{0}^{x} \frac{(x-s)^{n-3}}{(n-3) !} v_{u}(s) d s,
\end{aligned}
$$

with

$$
\begin{aligned}
v_{u}(x):= & \delta_{n-2}\left(0, u^{(n-2)}(0)+g_{n-2}\left(u, u^{\prime}, \ldots, u^{(n-2)}, u^{(n-2)}(0), u^{(n-1)}(0)\right)\right) \\
& +\int_{0}^{x} \varphi^{-1}\left(\tau_{u}-\int_{0}^{s} F_{u}(r) d r\right) d s
\end{aligned}
$$

and $\tau_{u} \in \mathbb{R}$ is the unique solution of the equation

$$
\begin{aligned}
\delta_{n-2}\left(1, u^{(n-2)}(1)+g_{n-1}\left(u, u^{\prime}, \ldots, u^{(n-2)}, u^{(n-2)}(1), u^{(n-1)}(1)\right)\right) & \\
-\delta_{n-2}\left(0, u^{(n-2)}(0)+g_{n-2}\left(u, u^{\prime}, \ldots, u^{(n-2)}, u^{(n-2)}(0), u^{(n-1)}(0)\right)\right) & =\int_{0}^{1} \varphi^{-1}\left(\tau_{u}-\int_{0}^{s} F_{u}(r) d r\right) d s .
\end{aligned}
$$

By (18), there is a function $\omega \in L^{1}(I)$ such that

$$
\left|F_{u}(s)\right| \leq \omega(s) \text { for a. e. } s \in I \text { and for all } u \in C^{n-1}(I) \text {; }
$$


and, by (24), there exists $L>0$ such that

$$
\left|\tau_{u}\right| \leq L \text { for all } u \in C^{n-1}(I)
$$

Hence, the operator $\mathcal{T}\left(C^{n-1}(I)\right)$ is bounded in $C^{n-1}(I)$, and by Schauder's fixed point theorem, the operator $\mathcal{T}$ has a fixed point $u_{1}$. If $n=2$, the proof is similar.

Step 3: $u_{1}(x)$ is a solution of problem (1)-(2).

This function $u_{1}(x)$ will be a solution of the initial problem (1)-(2) if the following inequalities hold

$$
\begin{aligned}
\alpha^{(i)}(0) & \leq u_{1}^{(i)}(0)+g_{i}\left(u_{1}, u_{1}^{\prime}, \ldots, u_{1}^{(n-2)}, u_{1}^{(i)}(0)\right) \\
& \leq \beta^{(i)}(0), \quad i=0, \ldots, n-3, \\
\alpha^{(n-2)}(0) & \leq u_{1}^{(n-2)}(0)+g_{n-2}\left(u_{1}, u_{1}^{\prime}, \ldots, u_{1}^{(n-2)}, u_{1}^{(n-2)}(0), u_{1}^{(n-1)}(0)\right) \\
& \leq \beta^{(n-2)}(0), \\
\alpha^{(n-2)}(1) & \leq u_{1}^{(n-2)}(1)+g_{n-1}\left(u_{1}, u_{1}^{\prime}, \ldots, u_{1}^{(n-2)}, u_{1}^{(n-2)}(1), u_{1}^{(n-1)}(1)\right) \\
& \leq \beta^{(n-2)}(1) .
\end{aligned}
$$

To prove the first one, assume for the sake of a contradiction, that there is an $i_{0}, 0 \leq i_{0} \leq$ $n-3$, such that

$$
u_{1}^{\left(i_{0}\right)}(0)+g_{i_{0}}\left(u_{1}, u_{1}^{\prime}, \ldots, u_{1}^{(n-2)}, u_{1}^{\left(i_{0}\right)}(0)\right)<\alpha^{\left(i_{0}\right)}(0)
$$

By (19), $u_{1}^{\left(i_{0}\right)}(0)=\alpha^{\left(i_{0}\right)}(0)$, and, by the monotone assumptions on the function $g_{i_{0}}$ and (11), we obtain

$$
\begin{aligned}
0 & >g_{i_{0}}\left(u_{1}, u_{1}^{\prime}, \ldots, u_{1}^{(n-2)}, u_{1}^{\left(i_{0}\right)}(0)\right)=g_{i_{0}}\left(u_{1}, u_{1}^{\prime}, \ldots, u_{1}^{(n-2)}, \alpha^{\left(i_{0}\right)}(0)\right) \\
& \geq g_{i_{0}}\left(\alpha, \alpha^{\prime}, \ldots, \alpha^{(n-2)}, \alpha^{\left(i_{0}\right)}(0)\right) \geq 0
\end{aligned}
$$


which is a contradiction. Thus, $\alpha^{(i)}(0) \leq u_{1}^{(i)}(0)+g_{i}\left(u_{1}, u_{1}^{\prime}, \ldots, u_{1}^{(n-2)}, u_{1}^{(i)}(0)\right)$ for every $i=0, \ldots, n-3$. In the same way, we can show that

$$
u_{1}^{(i)}(0)+g_{i}\left(u_{1}, u_{1}^{\prime}, \ldots, u_{1}^{(n-2)}, u_{1}^{(i)}(0)\right) \leq \beta^{(i)}(0)
$$

for each $i=0, \ldots, n-3$.

Suppose that the first inequality in (26) does not hold; then by $(19), u_{1}^{(n-2)}(0)=\alpha^{(n-2)}(0)$, and so $u_{1}^{(n-1)}(0) \geq \alpha^{(n-1)}(0)$ by (20). By the monotone assumptions on $g_{n-2}$ and (11), we obtain the contradiction

$$
\begin{aligned}
0 & >g_{n-2}\left(u_{1}, u_{1}^{\prime}, \ldots, u_{1}^{(n-2)}, u_{1}^{(n-2)}(0), u_{1}^{(n-1)}(0)\right) \\
& =g_{n-2}\left(u_{1}, u_{1}^{\prime}, \ldots, u_{1}^{(n-2)}, \alpha^{(n-2)}(0), u_{1}^{(n-1)}(0)\right) \\
& \geq g_{n-2}\left(\alpha, \alpha^{\prime}, \ldots, \alpha^{(n-2)}, \alpha^{(n-2)}(0), \alpha^{(n-1)}(0)\right) \geq 0 .
\end{aligned}
$$

Hence, $\alpha^{(n-2)}(0) \leq u_{1}^{(n-2)}(0)+g_{n-2}\left(u_{1}, u_{1}^{\prime}, \ldots, u_{1}^{(n-2)}, u_{1}^{(n-2)}(0), u_{1}^{(n-1)}(0)\right)$, and by the same method, it can be shown that

$$
u_{1}^{(n-2)}(0)+g_{n-2}\left(u_{1}, u_{1}^{\prime}, \ldots, u_{1}^{(n-2)}, u_{1}^{(n-2)}(0), u_{1}^{(n-1)}(0)\right) \leq \beta^{(n-2)}(0) .
$$

Assuming that

$$
\alpha^{(n-2)}(1)>u_{1}^{(n-2)}(1)+g_{n-1}\left(u_{1}, u_{1}^{\prime}, \ldots, u_{1}^{(n-2)}, u_{1}^{(n-2)}(1), u_{1}^{(n-1)}(1)\right)
$$

by the same type of arguments we have

$$
u_{1}^{(n-2)}(1)=\alpha^{(n-2)}(1) \text { and } u_{1}^{(n-1)}(1) \leq \alpha^{(n-1)}(1)
$$

Therefore, by the properties of $g_{n-1}$, we again arrive at the contradiction

$$
\begin{aligned}
0 & >g_{n-1}\left(u_{1}, u_{1}^{\prime}, \ldots, u_{1}^{(n-2)}, u_{1}^{(n-2)}(1), u_{1}^{(n-1)}(1)\right) \\
& =g_{n-1}\left(u_{1}, u_{1}^{\prime}, \ldots, u_{1}^{(n-2)}, \alpha^{(n-2)}(1), u_{1}^{(n-1)}(1)\right) \\
& \geq g_{n-1}\left(\alpha, \alpha^{\prime}, \ldots, \alpha^{(n-2)}, \alpha^{(n-2)}(1), \alpha^{(n-1)}(1)\right) \geq 0 .
\end{aligned}
$$


The remaining inequality can also be proved by the above technique.

\section{EXAMPles}

In this section, we present two examples to illustrate our results.

Example 1. Consider the differential equation

$$
-\left(\left(u^{(n-1)}(x)\right)^{3}\right)^{\prime}=(u(x)+1)^{3}+\left(u^{(n-3)}(x)\right)^{2 \theta+1}-d\left(u^{(n-2)}(x)\right)^{5}, \quad x \in(0,1),
$$

and the boundary conditions

$$
\begin{gathered}
u(0)=\frac{1}{b} \int_{0}^{x} u(s) d s, \\
u^{(i)}(0)=\frac{1}{c} \sum_{k=1}^{m} a_{i, k} u^{(i)}\left(\eta_{i, k}\right), \quad i=1, \ldots, n-2, \\
u^{(n-2)}(1)=\max _{x \in[0,1]} u^{(n-2)}(x),
\end{gathered}
$$

where $n \geq 3, \theta, m \in \mathbb{N}, b, c, d \in \mathbb{R}, a_{i, k} \geq 0$, for $i=1, \ldots, n-2$ and $k=1, \ldots, m, \sum_{k=1}^{m} a_{i, k} \leq 1$, $b \geq 2, c \geq 3, d \geq 3$, and for each $i=1, \ldots, n-2$, we have $0<\eta_{i, 1}<\ldots<\eta_{i, m} \leq 1$.

The problem (28)-(29) is a particular case of problem (1)-(2) with

$$
\begin{aligned}
\phi(z) & =z^{3}, \\
f\left(x, y_{0}, \ldots, y_{n-1}\right) & =\left(y_{0}+1\right)^{3}+\left(y_{n-3}\right)^{2 \theta+1}-d\left(y_{n-2}\right)^{5}, \\
g_{0}\left(y_{0}, \ldots, y_{n-1}\right) & =\frac{1}{b} \int_{0}^{x} y_{0}(s) d s-y_{n-1} \\
g_{i}\left(y_{0}, \ldots, y_{n-1}\right) & =\frac{1}{c} \sum_{k=1}^{m} a_{i, k} y_{i}\left(\eta_{i, k}\right)-y_{n-1}, \quad i=1, \ldots, n-3, \\
g_{n-2}\left(y_{0}, \ldots, y_{n-1}, y_{n}\right) & =\frac{1}{c} \sum_{k=1}^{m} a_{n-2, k} y_{n-2}\left(\eta_{n-2, k}\right)-y_{n-1}, \\
g_{n-1}\left(y_{0}, \ldots, y_{n-1}, y_{n}\right) & =\max _{x \in I} y_{n-2}(x)-y_{n-1} .
\end{aligned}
$$


The functions $\alpha, \beta: \mathbb{R} \rightarrow \mathbb{R}$ given by

$$
\alpha(x)=-\sum_{k=0}^{n-2} \frac{x^{k}}{k !} \quad \text { and } \quad \beta(x)=\sum_{k=0}^{n-2} \frac{x^{k}}{k !}
$$

are, respectively, lower and upper solutions of (28)-(29). Since $f$ does not depend on the derivative $u^{(n-1)}$, it satisfies the Nagumo-type growth condition in every bounded set $E \subseteq$ $I \times \mathbb{R}^{n}$. Hence, by Theorem 6, there is a nontrivial solution $u$ of problem (28)-(29) such that

$$
\alpha^{(i)}(x) \leq u^{(i)}(x) \leq \beta^{(i)}(x)
$$

for every $x \in I$ and $i=0, \ldots, n-2$. Moreover, by the location part, this solution $u$ can not be polynomial of order greater than $n-2$. For example, if $n=4$, this solution can not be a cubic function.

In our final example, we have $\phi(\mathbb{R}) \neq \mathbb{R}$.

Example 2. Consider the boundary value problem

$$
\begin{gathered}
-\left(\phi\left(u^{(n-1)}(x)\right)\right)^{\prime}=(u(x))^{3}-2\left(u^{(n-2)}(x)\right)^{2 m-1}+\left|u^{(n-1)}(x)\right|^{\theta}, \\
u^{(i)}(0)=0, \quad i=0, \ldots, n-3, \\
u^{(n-2)}(0)=\max _{x \in I} u^{(n-2)}(x)+\left(u^{(n-1)}(0)\right)^{3} \\
u^{(n-2)}(1)=\frac{1}{b} \sum_{i=0}^{n-2} u^{(i)}\left(\eta_{i}\right)-u^{(n-1)}(1)+k
\end{gathered}
$$

where

$$
\phi(x)= \begin{cases}\arctan (x-6)+216, & x>6, \\ x^{3}, & -6 \leq x \leq 6, \\ \arctan (x+6)-216, & x<-6,\end{cases}
$$


$n \geq 3, m \in \mathbb{N}, 0 \leq \theta \leq 2, b>n-1,0 \leq \eta_{i} \leq 1$, for $i=0, \ldots, n-2$, and $k \in \mathbb{R}$ with $k \in\left[-1+\frac{n-1}{b}, 1-\frac{n-1}{b}\right]$. Clearly, this is a particular case of problem (1)-(2) with

$$
\begin{aligned}
f\left(x, y_{0}, \ldots, y_{n-1}\right) & =\left(y_{0}\right)^{3}-2\left(y_{n-2}\right)^{2 m-1}+\left|y_{n-1}\right|^{\theta}, \\
g_{i}\left(y_{0}, \ldots, y_{n-1}\right) & =y_{n-1}, \quad i=0, \ldots, n-3, \\
g_{n-2}\left(y_{0}, \ldots, y_{n-1}, y_{n}\right) & =\max _{x \in I} y_{n-2}(x)+\left(y_{n}\right)^{3}-y_{n-1}, \\
g_{n-1}\left(y_{0}, \ldots, y_{n-1}, y_{n}\right) & =\frac{1}{b} \sum_{i=0}^{n-2} y_{i}\left(\eta_{i}\right)-y_{n}-y_{n-1}+k,
\end{aligned}
$$

Note that existence results for the $\phi$-Laplacian with the assumption $\phi(\mathbb{R})=\mathbb{R}$, are not applicable to equation (30). The polynomial functions $\alpha, \beta: \mathbb{R} \rightarrow \mathbb{R}$ given by

$$
\alpha(x)=-\frac{x^{n-2}}{(n-2) !} \quad \text { and } \quad \beta(x)=\frac{x^{n-2}}{(n-2) !}
$$

are, respectively, lower and upper solutions of (30)-(31). We see that $f$ is a $L^{1}$-Carathéodory function and satisfies the Nagumo-type growth condition in the set

$$
E_{*}:=\left\{\begin{array}{c}
\left(x, y_{0}, \ldots, y_{n-1}\right) \in[0,1] \times \mathbb{R}^{n}:-\frac{x^{n-2}}{(n-2) !} \leq y_{0} \leq \frac{x^{n-2}}{(n-2) !} \\
-x \leq y_{n-3} \leq x, \quad-1 \leq y_{n-2} \leq 1
\end{array}\right\}
$$

with $h_{E}\left(\left|y_{n-1}\right|\right):=3+\left|y_{n-1}\right|^{\theta}$. In fact, it is easy to see that $r=2$. If we choose $R=3$, then

$$
\int_{\phi(r)}^{\phi(R)} \frac{\left|\phi^{-1}(s)\right|}{h_{E}\left(\left|\phi^{-1}(s)\right|\right)} d s=\int_{8}^{27} \frac{s^{\frac{1}{3}}}{3+s^{\frac{\theta}{3}}} d s \geq \int_{8}^{27} \frac{2}{3+3^{\theta}} d s>2
$$

since $0 \leq \theta \leq 2$. Therefore, by Theorem 6 , there is a solution $u$ of the problem (30)-(31) such that

$$
\alpha^{(i)}(x) \leq u^{(i)}(x) \leq \beta^{(i)}(x)
$$

for every $x \in[0,1]$ and $i=0, \ldots, n-2$. If $k \neq 0$, the solution is nontrivial. Moreover, by the location part, this solution $u$ can not be a polynomial of degree greater than $n-2$. For example, if $n=4$, this solution can not be a cubic function. Finally, we observe that if 
$\eta_{i}=0$ for all $i=0, \ldots, n-2$, then in the last equation in boundary condition (31) we have

$$
\frac{1}{b} \sum_{i=0}^{n-2} u^{(i)}\left(\eta_{i}\right)=\frac{1}{b} u^{(n-2)}(0) .
$$

Hence, we need $k \in\left[-1+\frac{1}{b}, 1-\frac{1}{b}\right]$ to ensure that $\alpha$ and $\beta$ are lower and upper solutions. Note that in this case $k$ does not depend on $n$.

\section{REFERENCES}

[1] A. Cabada and F. Minhós, Fully nonlinear fourth order equations with functional boundary conditions, J. Math. Anal. Appl. 340 (2008), 239-251.

[2] A. Cabada, F. Minhós, and A. I. Santos, Solvability for a third order discontinuous fully equation with functional boundary conditions, J. Math. Anal. Appl. 322 (2006) ,735-748.

[3] A. Cabada, R. Pouso, and F. Minhós, Extremal solutions to fourth-order functional boundary value problems including multipoint conditions, Nonlinear Anal.: Real World Appl. 10 (2009), 2157-2170.

[4] J. R. Graef and L. Kong, Solutions of second order multi-point boundary value problems, Math. Proc. Camb. Phil. Soc. 145 (2008), 489-510.

[5] J. R. Graef, L. Kong, and Q. Kong, Higher order multi-point boundary value problems, Math. Nachr., to appear.

[6] J. R. Graef, L. Kong, and F. Minhós, Higher order $\phi$-Laplacian BVP with generalized Sturm-Liouville boundary conditions, to appear.

[7] J. R. Graef, L. Kong, and B. Yang, Existence of solutions for a higher order multi-point boundary value problems, Result. Math. 53 (2009), 77-101.

[8] M. R. Grossinho, F. Minhós, and A. I. Santos, A note on a class of problems for a higher order fully nonlinear equation under one sided Nagumo type condition, Nonlinear Anal. 70 (2009) 4027-4038

[9] J. Henderson, Solutions of multipoint boundary value problems for second order equations, Dynam. Systems Appl. 15 (2006), 111-117.

[10] J. Henderson and S. K. Ntouyas, Positive solutions for systems of nth order three-point nonlocal boundary value problems, Electron. J. Qual. Theory Differ. Equ. (2007), No. 18, pp. 1-12.

[11] F. Minhós, On some third order nonlinear boundary value problems: existence, location and multiplicity results, J. Math. Anal. Appl. 339 (2008), 1342-1353.

[12] F. Minhós, and J. Fialho, On the solvability of some fourth-order equations with functional boundary conditions, Discrete Contin. Dyn. Syst. 2009, suppl., to appear.

[13] M. Wang, A. Cabada, and J. J. Nieto, Monotone method for nonlinear second order periodic boundary value problems with Carathéodory functions, Ann. Polon. Math. 58 (1993), 3, 221-235.

[14] X. Zhang and L. Liu, Nontrivial solutions for higher order multi-point boundary value problems, Comput. Math. Appl. 56 (2008), 861-873. 
Department of Mathematics, University of Tennessee at Chattanooga, Chattanooga, Tn 37403 , USA.

E-mail address: John-Graef@utc.edu

Department of Mathematics, University of Tennessee at Chattanooga, Chattanooga, Tn 37403 , USA.

E-mail address: Lingju-Kong@utc.edu

Department of Mathematics, University of Évora Research Centre on Mathematics and its Applications (CIMA-UE), Rua Romäo Ramalho, 59 7000-671 Évora, Portugal

E-mail address: fminhos@uevora.pt 